\title{
Ancestral mental number lines: What is the evidence?
}

\author{
Rafael Núñez \\ Department of Cognitive Science \\ University of California, San Diego \\ 9500 Gilman Drive, La Jolla, CA 92093-0515, U.S.A.
}

Wim Fias

Department of Experimental Psychology

Ghent University

H. Dunantlaan 2, B-9000 Ghent, Belgium

to be published as: Nunez, R., \& Fias, W. (201?).

Ancestral mental number lines: What is the

evidence? Cognitive Science. DOI: 10.1111/cogs.

12296

Corresponding author: Núñez, R (rnunez@ucsd.edu)

Keywords: number-to-space mappings, mental number line, number cognition, association biases, brain asymmetry, lateralization, learning, culture 


\begin{abstract}
Some developmental, cross-cultural, and comparative studies have suggested that number-to-space mappings are biologically endowed universals. Going further, a recent study has claimed that newborn chicks map numbers to space, resembling humans' mental number line. The data in these studies, however, derive from loose operational definitions and don't provide evidence of numerosity-to-space mappings, let alone of mental number lines. Regarding newborn chicks, crucial baseline information involving spontaneous lateralized behavior has been overlooked. Even when downgrading claims from number line mappings to mere biases in numerosity-to-space associations, results can be explained independently of them via lateralized processing in chicks' brains. We suggest some experiments to address outstanding questions.
\end{abstract}


Over the last two decades substantial efforts have been dedicated to investigate the question of whether the building blocks of human mathematical concepts ultimately have their origins in biological evolution. A relevant case study is the 'mental number line' hypothesis, which states that numbers are represented in the brain as spatial entities along a mental line, yielding behavioral manifestations. Some developmental (de Hevia and Spelke, 2009, 2010), cross-cultural (Dehaene, Izard, Spelke, and Pica, 2008a), and comparative (Drucker and Brannon, 2014) studies have suggested that number-to-space mappings - underlying mental number lines - are biologically endowed universals, emerging independently of language and culture. Recently, going further, Rugani, Vallortigara, Priftis, and Regolin (2015) have argued that newborn domestic chicks (Gallus gallus) map numbers to space resembling humans' mental number line, and claimed that "spatial mapping of numbers from left to right may be a universal cognitive strategy available soon after birth" (p. 536). After training newborn chicks to circumnavigate a centered panel depicting a target numerosity (5 elements for some chicks, 20 for others), the researchers allowed the chicks to explore an environment containing two panels - to the left and to the right, displaying identical numerosities either smaller or greater than the target ( 2 or 8 elements, and 8 or 32 , respectively). The authors reported that around $70 \%$ of the time the chicks preferred the left panel when the numerosity was smaller than the target and the right one when it was greater. They interpreted these results as evidence that there is a left-to-right number-to-space mapping in newborn chicks that resembles humans' mental number line. But, do the data really support these claims? 
Basic criteria for number line mappings must be satisfied

When testing for a number line mapping, construct validity requires that the study's operational definitions allow for establishing (a) the presence of a number-space mapping, (b) applying over a line, and (c) exhibiting a metric space-i.e., with a distance function. Any standard dictionary or encyclopedic definition of mapping (e.g., "an operation that associates each element of a given set (the domain) with one or more elements of a second set (the range)" (Stevenson \& Lindberg, 2010); "any prescribed way of assigning to each object in one set a particular object in another (or the same) set" (Encyclopædia Britannica, 2015); our italics) tells that the chicks' mere preference of one of two identical panels does not constitute a mapping (criterion a). In order to establish the presence of a proper mapping, the study should show that chicks, after being randomly presented various numerosities, select specific locations in space for each of them.

Similar inadequate operationalizations of "mapping" are present in developmental and comparative studies that argue for a purely biologically determined number-tospace mappings in children and non-human animals-relying simply on associations indexed by dishabituation looking time (de Hevia and Spelke, 2010), by biases in bisection tasks (de Hevia and Spelke, 2009), or that follow training with purely spatial arrays (Drucker and Brannon, 2014). These loose operationalizations are misleading as they yield conclusions that obscure the seemingly fundamental role of learning and cultural factors in the constitution of genuine (cardinal) number-to-space mappings. 
Historical data, after all, indicate that the number line was only invented in the $17^{\text {th }}$ century via the pioneering mathematical work of Napier (1614/1616) and Wallis (1685), after centuries of conceptual struggles and development (Núñez, 2011).

Importantly, even if Rugani et al.'s (2015) study were able to establish the presence of a proper mapping, it would then need to show that the mapping is performed along a line (criterion b), and crucially, exhibiting a metric space (criterion c). The study does establish the relative response to 8 elements with respect to small ( 5 , right) and large targets (20, left), but it only does it with different groups of chicks, and by just considering left-right binary choices based on two numerosity stimuli. Thus, the crucial distance-function criterion (c) of the number line is left unsatisfied. This missing component is highly relevant, as even in human groups without writing practices and formal schooling, a bi-categorical number/numerosity-to-line mapping can be manifested in the absence of a spontaneous mapping exhibiting a distance function (criterion c). Dehaene, Izard, Spelke, and Pica (2008b) observed (but did not analyze) that among the Mundurukú of the Amazon a (statistically) significant number of experimental runs in their study lacked a distance function, exhibiting bi-categorical mappings that primarily used the line's endpoints but not its intermediate locations, a fact that, ironically, goes against their conclusion that the intuition of mapping numbers to a line is spontaneous and universal (Dehaene et al., 2008a). Similarly, unschooled Yupno of Papua New Guinea, despite having a number lexicon and a cardinal understanding of number concepts beyond ten, exhibited this bi-categorical mapping with no distance function in virtually all tested mapping trials, across the entire set of 
stimuli- symbolic and non-symbolic (Núñez, Cooperrider, and Wassmann, 2012).

In short, to establish the presence of a proper number line mapping all three criteria above must be satisfied. Rugani et al. (2015) did not do this.

\section{Baseline behaviors must be well established}

Even when downgrading the claims from number-to-space mappings with metric to mere biases in numerosity-space associations, Rugani et al.'s data (2015) permit an alternative explanation. Indeed, the observed responses might emanate from asymmetries in the chicks' brain. Due to the absence of a corpus callosum in chicks, hemispheric differences in the processing of information are magnified in behavior, with specific and systematic effects on spatial action, such as search-driven lateralized head turning and right-hemisphere-lateralized reaction to novelty (Vallortigara, Regolin, Bortolomiol, and Tommasi, 1996; Tommasi, Andrew, and Vallortigara, 2000) which result in a left-biased exploration behavior (Vallortigara and Andrew,1991; Andrew and Rogers, 2002). Such biases call for a detailed investigation of baseline responses in the experimental paradigm, and/or, at the very least, testing against null hypotheses that take into account these known biases (i.e., not just testing against a $50 \%$ chance of producing a left or right response, as Rugani et al. (2015) did). In fact, when proper statistical analyses are performed, results cast serious doubts on the conclusions of the study (Harshaw, in press).

Rugani et al.'s (2015) paradigm critically depends on presenting novel test stimuli relative to a trained target. Given that spatial choice appears to be modulated by target- 
relative numerosity size, left-right hemisphere differences that systematically covary with the chosen stimuli -e.g., processing of novelty - should be examined meticulously. The authors made substantial efforts to control for several possible confounds. It remains to be considered, however, that numerosity is neurally coded in a logarithmiclike compressed manner, which according to single-cell neurophysiology holds for the two types of neural coding known to underlie numerosity: (i) numerosity-selective coding for which the tuning width of numerosity-selective cells increases with increasing numerosity (Nieder, Freedman, and Miller, 2002), and (ii) accumulation coding (Roitman, Brannon, and Platt, 2007; Stoianov and Zorzi, 2012) for which the amount of neural acitivity corresponds to numerosity. Although there are no neural data about numerosity coding in birds, an overwhelming number of studies have reported behavioral signatures consistent with non-linear compressive coding across a wide variety of species, including birds (Dehaene, Dehaene-Lambertz, and Cohen, 1998). Because of this logarithmic-like compressive coding, small-to-target numerosity contrasts are more pronounced than large-to-target contrasts, making the former more discriminable and thus more novel than the latter. Given the asymmetrical brain response to novelty, the two hemispheres may have contributed differently to the chicks' choice when confronted with small-to-target or large-to-target numerosity contrasts. This asymmetrical brain response to novelty may well have interacted with other hemispheric differences driving the chicks' lateralized behavior (Andrew and Rogers, 2002). In the absence of a proper baseline condition, however, this is impossible to pinpoint. Which choice do chicks spontaneously make without a trained numerosity target? And when 
presented the same numerosity as the target? Or when presented stimuli that do not involve numerosity at all?

\section{Conclusion}

In sum, further evidence - with proper operationalizations - is needed to support the strong claims in developmental (de Hevia and Spelke, 2009, 2010) and comparative studies (Drucker and Brannon, 2014; Rugani et al, 2015) that number-to-space mappings are biologically endowed universals. To test a spontaneous numerosity-tospace association in chicks a study should be conducted without a numerosity target, controlling for novelty and other confounds, and testing for relative numerosity contrasts within - not between individuals, as Rugani et al. (2015) did. If the numerosity-to-space association can be solidly established, then the next challenge would be to show that these association biases actually constitute numerosity-to-space mappings exhibiting a distance-function, which then ultimately may resemble the (schooled) human's mental number line.

\section{References}

Andrew, R., \& Rogers, L. (2002). The nature of lateralization in tetrapods. In L. Rogers \& R. Andrew (Eds.), Comparative vertebrate lateralization (pp. 94-125). Cambridge, UK: Cambridge University Press.

Dehaene, S., Dehaene-Lambertz, G., \& Cohen, L. (1998). Abstract representation of numbers in the animal and human brain. Trends in Neurosciences, 21, 355-361. 
Dehaene S, Izard V, Spelke E, Pica P. (2008a). Log or linear? Distinct intuitions of the number scale in Western and Amazonian indigene cultures. Science, 320, 12171220.

Dehaene, S., Izard, V., Spelke, E., \& Pica, P. (2008b). Supplementary material to "Log or linear? Distinct intuitions of the number scale in Western and Amazonian indigene cultures." Science online www.sciencemag.org/cgi/content/full/320/5880/1217/DC1.

de Hevia, M. D., Spelke, E. (2009). Spontaneous mapping of number and space in adults and young children. Cognition, 110, 198-207.

de Hevia, M. D., Spelke, E. (2010). Number-space mappings in human infants.

Psychological Science, 21, 653-660.

Drucker, C. \& Brannon, E. (2014). Rhesus monkeys (Macaca mulatta) map number onto space. Cognition, 132, 57-67.

Encyclopædia Britannica website (2015). Available:

http://www.britannica.com/EBchecked/topic/363594/mapping. Accessed 2015 February 27.

Harshaw, C. (in press). Comment on "Number-space mapping in the newborn chick resembles humans' mental number line." Science.

Napier, J. (1614/1616). A description of the admirable table of logarithmes (English translation from Latin by E. Wright). London: N. Oakes.

Nieder, A., Freedman, D. J., \& Miller, E. K. (2002). Representation of the quantity of visual items in the primate prefrontal cortex. Science, 297, 1708-1711. 
Núñez, R. (2011). No innate number line in the human brain. Journal of Cross-Cultural Psychology, 42(4), 651-668.

Núñez, R., Cooperrider, K., \& Wassmann, J. (2012). Number Concepts without Number Lines in an Indigenous Group of Papua New Guinea. PLOS ONE. DOI:

10.1371/journal.pone.0035662

Roitman, J. D., Brannon, E. M., \& Platt, M. L. (2007). Monotonic Coding of Numerosity in Macaque Lateral Intraparietal Area. Plos Biology, 5(8), e208. DOI:

10.1371/journal.pbio.0050208

Rugani, R., Vallortigara, G., Priftis, K., and Regolin, L. (2015). Number-space mapping in the newborn chick resembles humans' mental number line. Science, 347, 534536.

Stevenson, A. \& Lindberg, C.A. (Eds.) (2010). New Oxford American Dictionary (3 ${ }^{\text {rd }}$ edition). New York: Oxford University Press.

Stoianov, I., \& Zorzi, M. (2012). Emergence of a "visual number sense" in hierarchical generative models. Nature Neuroscience, 15, 194-196.

Tommasi, L., Andrew, R.J., and Vallortigara, G. (2000). Eye use in search is determined by the nature of task in the domestic chick (Gallus gallus). Behavioural Brain Research, 112, 119-126.

Vallortigara G. \& Andrew RJ. (1991). Lateralization of response by chicks to change in a model partner. Animal Behavior, 41, 187-194. 
Vallortigara G, Regolin L, Bortolomiol G, Tommasi L. (1996). Lateral asymmetries due to preferences in eye use during visual discrimination learning in chicks. Behavioural Brain Research, 74, $135-43$.

Wallis, J. (1685). A treatise of algebra. London: John Playford. 